\title{
Initiation of Polymerization of Vinyl Monomers by Charge Transfer Mechanism
}

\author{
N. N. DASS and S. S. BEGUM* \\ Department of Chemistry, Dibrugarh University, \\ Dibrugarh-786004, Assam, India \\ *Institute of Pharmacy, Assam Medical College, \\ Dibrugarh-786002, Assam, India
}

(Received January 30, 1987)

\begin{abstract}
The charge transfer initiated polymerization of acrylonitrile by 2,4-dinitrophenylhydrazine (DNPH) and $\mathrm{Cu}^{2+}$ in the presence of carbon tetrahalide was studied in dimethyl sulphoxide at $60^{\circ} \mathrm{C}$. The rate of polymerization became maximum when the mol ratio of [DNPH] to $\left[\mathrm{Cu}^{2+}\right]$ was $4: 1$. The polymerization was inhibited after a very short period when the above ratio was $2: 1$, mainly due to the liberated bromine. A suitable mechanism for the experimental observation is proposed.

KEY WORDS Charge Transfer Polymerization / Acrylonitrile / 2,4Dinitrophenylhydrazine / Dimethyl Sulphoxide / Carbon Tetrabromide / Tetrakis Dimethyl Sulphoxide Copper(II) Perchlorate / Association of Bromine with Polyacrylonitrile /
\end{abstract}

Our previous works ${ }^{1-7}$ reveal that compounds containing a donor nitrogen atom can play a significant role in initiation and propagation steps of charge transfer (CT) initiated polymerization. The CT initiated polymerization of vinyl monomers like methyl methacrylate (MMA), ${ }^{1-7}$ 2-vinylpyridine, ${ }^{8}$ etc. has already been reported. It was found that metal ions have profound effect on the rate of $\mathrm{CT}$ initiated polymerization. It should be worthwhile to follow the rate of polymerization of acrylonitrile (AN) in the presence of metal ions and carbon tetrahalide for elucidating this complex but important problem. The present communication reports the polymerization of $\mathrm{AN}$ in the presence of 2,4-dinitrophenylhydrazine (DNPH) and tetrakis dimethyl sulphoxide copper(II) perchlorate, $\left[\mathrm{Cu}(\mathrm{DMSO})_{4}\right]\left(\mathrm{ClO}_{4}\right)_{2}, \mathrm{~A}$ with carbon tetrahalides in dimethyl sulphoxide (DMSO) at $60^{\circ} \mathrm{C}$.

\section{EXPERIMENTAL}

\section{Materials}

$\mathrm{AN},{ }^{9} \mathrm{DMSO}^{5}$ and carbon tetrachloride ${ }^{5}$ were purified as reported in the literature. Carbon tetrabromide and DNPH were used without further purification.

The complex, A, was prepared as before. ${ }^{5}$

\section{Polymerization}

Experiments were done in a three limbed vessel and the rates of polymerization were measured dilatometrically in the usual way. ${ }^{10}$

\section{Molecular Weight Determination}

Polymers were washed with methanol and dried after precipitation with water. The weight average molecular weights of AN polymers were determined viscometrically in $N, N$-dimethylformamide at $25^{\circ} \mathrm{C}$. The following relation was used in the calculation. 


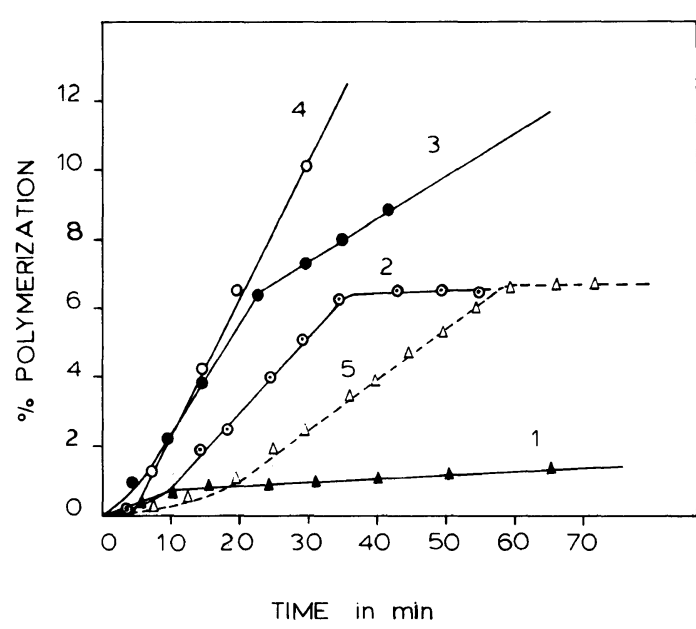

Figure 1. Polymerization of $A N$ in DMSO initiated by $\mathrm{DNPH}$ and $\mathrm{CBr}_{4}$ in the presence of $\mathrm{A}$ at $60^{\circ} \mathrm{C}$. [AN] $=$ $2.92 \mathrm{moll}^{-1} ;\left[\mathrm{CBr}_{4}\right]=1 \times 10^{-2} \mathrm{moll}^{-1} ;[\mathrm{A}]=2 \times 10^{-3}$ $\mathrm{moll}^{-1} ;[\mathrm{DNPH}]=2 \times 10^{-3} \mathrm{moll}^{-1}$ for curve $1,4 \times 10^{-3}$ moll $1^{-1}$ for curve $2,6 \times 10^{-3} \mathrm{moll}^{-1}$ for curve 3 , $8 \times 10^{-3} \mathrm{moll}^{-1}$ for curve 4 and $[\mathrm{KBr}]=1 \times 10^{-2}$ moll $1^{-1}$ for curve 5 .

$$
[\eta]=3.46 \times 10^{-4}\left(\bar{M}_{w}\right)^{0.733}
$$

Molecular weights of PMMA was determined with a Wescan 232A osmometer.

\section{RESULTS AND DISCUSSION}

A series of experiments were done by adding different amounts of DNPH to a constant composition of $\mathrm{AN}, \mathrm{A}$, and $\mathrm{CBr}_{4}$ in $\mathrm{DMSO}$ at $60{ }^{\circ} \mathrm{C}$. A few experimental curves are shown in Figure 1. Similar experiments were done using $\mathrm{CCl}_{4}$ in place of $\mathrm{CBr}_{4}$ (Figure 2). Experimentally it was found that the CT initiated polymerization of AN with DNPH alone was too slow. However, in the presence of a Lewis acid, like $\mathrm{Cu}^{2+}$, accelerated rate was observed. In all the cases, a very short induction period was recorded indicating that during induction period $\mathrm{Cu}^{2+}$ should be reduced to $\mathrm{Cu}^{1+}$ due to electron transfer ${ }^{12-14}$ from the primary radical to $\mathrm{Cu}^{2+}$. The rate of polymerization attained its maximum value when the mol ratio of DNPH to A was $4: 1$ in both the cases (Figures 1 and 2). Rate of

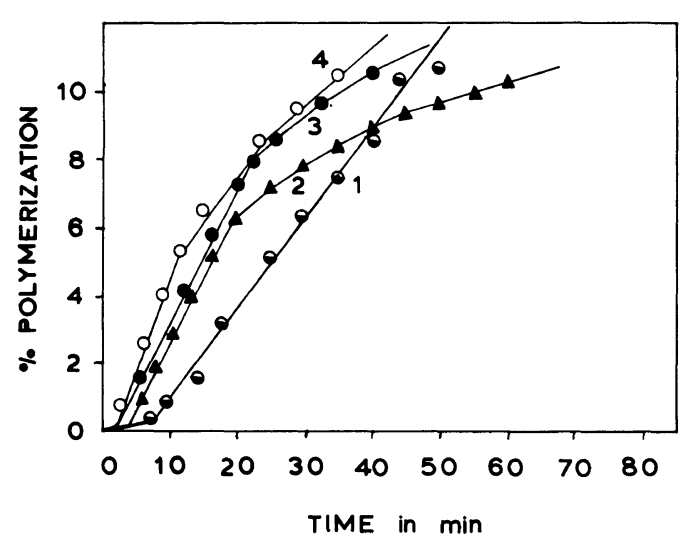

Figure 2. Polymerization of AN in DMSO initiated by $\mathrm{DNPH}$ and $\mathrm{CCl}_{4}$ in the presence of $\mathrm{A}$ at $60^{\circ} \mathrm{C}$. [AN] $=$ $2.92 \mathrm{moll}^{-1} ; \quad\left[\mathrm{CCl}_{4}\right]=1.04 \mathrm{moll}^{-1} ; \quad[\mathrm{A}]=2 \times 10^{-3}$ moll $1^{-1}$; [DNPH] $=1.2 \times 10^{-2} \mathrm{moll}^{-1}$ for curve 1 , $4 \times 10^{-3} \mathrm{moll}^{-1}$ for curve $2,6 \times 10^{-3} \mathrm{moll}^{-1}$ for curve $3,8 \times 10^{-3} \mathrm{moll}^{-1}$ for curve 4 .

polymerization decreased above this ratio due to the excess DNPH. Therefore, $[\mathrm{Cu}-$ $\left.(\mathrm{DNPH})_{4}\right]^{2+}$ is an accelerator. The following reaction scheme may explain the accelerated rate of polymerization of $\mathrm{AN}$ in presence of $\mathrm{DNPH}, \mathrm{A}$ and $\mathrm{CBr}_{4}$.

$$
4(\mathrm{DNPH})+\mathrm{Cu}^{2+} \underset{k_{2}}{\stackrel{k_{1}}{\rightleftharpoons}}\left[\mathrm{Cu}(\mathrm{DNPH})_{4}\right]^{2+}
$$

C

The complex $\mathbf{C}$, formed in situ will have unfavorable charge distribution around the metal ion. Therefore, a CT complex (II) will be formed in between $\mathbf{C}$ and $\mathrm{AN}$ in presence of $\mathrm{CBr}_{4}$ which readily decomposes to form the primary radical $R$.

$$
\begin{gathered}
{\left[\mathrm{Cu}(\mathrm{DNPH})_{4}\right]^{2+}+\mathrm{H}_{2} \mathrm{C}=\underset{\mathrm{H}}{\mathrm{C}}-\mathrm{CN}+\mathrm{CBr}_{4} \stackrel{k_{3}}{\longrightarrow} \mathrm{II}} \\
\mathrm{II} \stackrel{k_{4}}{\longrightarrow} \mathrm{Br}_{3} \mathrm{C}-\mathrm{CH}_{2}-\underset{\mathrm{C}^{+}}{\dot{\mathrm{C}}}-\mathrm{CN}+\mathrm{Br}^{-} \\
+\left[\mathrm{Cu}(\mathrm{DNPH})_{4}\right]^{+}
\end{gathered}
$$

The weak charge-transfer complex formed between $\mathrm{AN}$ and DNPH in presence of $\mathrm{CBr}_{4}$ 
Table I. Molecular weight of PAN, determined viscometrically

\begin{tabular}{ccc}
\hline$\frac{[\mathrm{DNPH}]}{\mathrm{mol1}{ }^{-1}}$ & Ratio [DNPH] to [A] & $\begin{array}{c}\text { Molecular } \\
\text { weight }\end{array}$ \\
\hline $4 \times 10^{-3}$ & $2: 1$ & $1.7 \times 10^{3}$ \\
$8 \times 10^{-3}$ & $4: 1$ & $6.2 \times 10^{3}$ \\
$1.2 \times 10^{-2}$ & $6: 1$ & $2.6 \times 10^{3}$ \\
\hline
\end{tabular}

Time of reaction, $1.5 \mathrm{~h}$

could not initiate the polymerization of AN. The nitrogen atom in the unsubstituted hydrazine group of DNPH acts as the donor atom. ${ }^{3}$ Due to the electron withdrawing inductive effect of the benzene ring, the free electrons in the nitrogen atom of the amine group of DNPH are less available than those of an aliphatic amine which alone can initiate the polymerization. ${ }^{1}$ Lewis acids are good electron acceptors and so $\mathrm{Cu}^{2+}$ increased the electron donating capacity of DNPH resulting in the rapid formation of free radicals.

The rate of polymerization with [DNPH] : $\left[\mathrm{Cu}^{2+}\right]$ greater than $4: 1$ was found to fall with the increase of added DNPH. The retarding effect of free DNPH is obvious since it is an aromatic nitro compound.

The molecular weight of the polymer formed was highest when the ratio of [DNPH] to [A] was 4:1. Some of the data for molecular weight of the polymer formed with different ratios of $[\mathrm{DNPH}]$ to $[\mathrm{A}]$ are quoted in Table I.

An interesting phenomenon occurred when the mol ratio of $[\mathrm{DNPH}]$ to $[\mathrm{A}]$ was $2: 1$. Here, the reaction was inhibited after a certain period. The reaction time decreased with the increase in the amount of DNPH with a fixed ratio of $2: 1$ of [DNPH] to [A]. It may be due to $\mathrm{CBr}_{4}$, liberated DNPH and consumption of primary radical for reduction of $\mathrm{Cu}^{2+}$. Since inhibition was more significant in the system with $\mathrm{CBr}_{4}$, we added $\mathrm{KBr}$ to it (Figure 1). The addition of $\mathrm{KBr}$ reduced the rate and increased the reaction time for polymerization as well as the induction period.

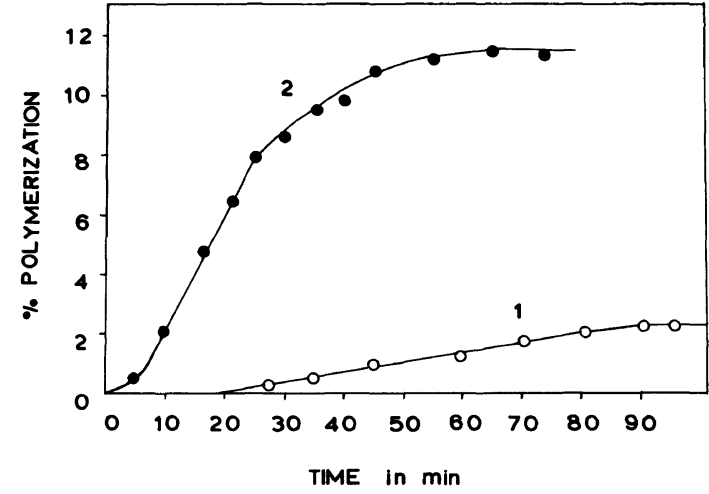

Figure 3. Polymerization of MMA in DMSO initiated by $\mathrm{DNPH}$ and $\mathrm{CBr}_{4}$ in the presence of $\mathrm{A}$ at $60^{\circ} \mathrm{C}$. [MMA] $=1.73 \mathrm{moll}^{-1} ;\left[\mathrm{CBr}_{4}\right]=1 \times 10^{-2} \mathrm{moll}^{-1} ;[\mathrm{A}]=$ $2 \times 10^{-3} \mathrm{moll}^{-1} ;[\mathrm{DNPH}]=4 \times 10^{-3} \mathrm{moll}^{-1}$ for curve 1 and $8 \times 10^{-3} \mathrm{moll}^{-1}$ for curve 2 .

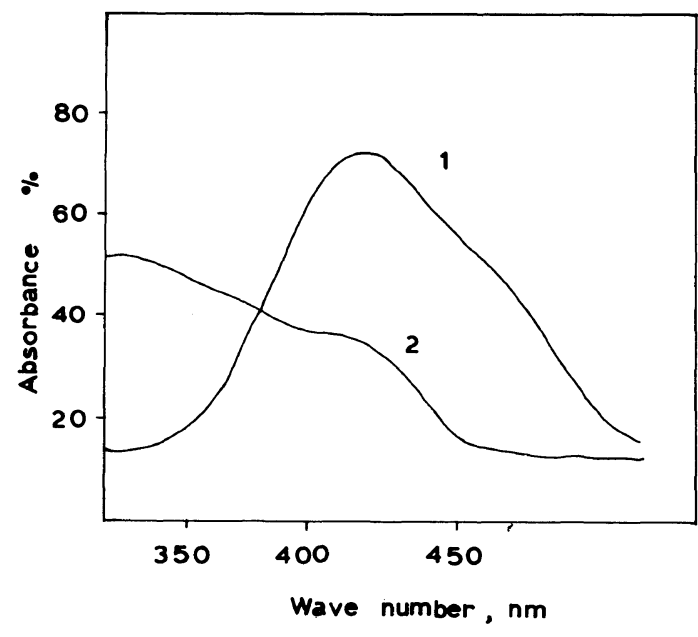

Figure 4. UV spectra of poly(MMA) in $\mathrm{CCl}_{4}$. (1) $\mathrm{Br}_{2}$ in $\mathrm{CCl}_{4}$; (2) poly(MMA) in $\mathrm{CCl}_{4}$.

But addition of $\mathrm{KBr}$ alone did not effect the rate of polymerization in the absence of $\mathrm{Cu}^{2+}$, suggesting $\mathrm{CuBr}_{2}$ acted as a retarder. But if $\mathrm{CuBr}_{2}$ is the only inhibiting species, the reaction time would have decreased drastically which was not experimentally observed.

The presence of DNPH in polyacrylonitrile (PAN) was difficult to establish by microanalysis, since the monomer itself contains nitrogen and IR analysis is not very sensitive to the end group present in the long chain. 
Though the quantitative isolation of pure PAN is possible, the quantitative estimation of DNPH was not possible since it complexed with $\mathrm{Cu}^{2+}$ in solution. The estimation of uncomplexed DNPH was also alluding since it involved a number of repeated steps to get the pure solid DNPH from the reaction mixture. So, some of the experiments were repeated replacing AN by MMA (Figure 3). The polymerization was inhibited after a short period even when MMA was used in place on $\mathrm{AN}$ and colored polymers of MMA with low molecular weight $\left(3.6 \times 10^{3}\right)$ were obtained, soluble even in alcohol and carbon tetrachloride. However, the polymer can be recovered from alcohol solution indicating its low solubility in alcohol. The presence of any end group containing nitrogen or halogen could not be established by IR analysis of the polymer. But the UV spectra of the polymer in $\mathrm{CCl}_{4}$ showed the presence of bromine. The appearance of a peak at $417 \mathrm{~nm}$ in the UV region for the polymer solution in carbon tetrachloride indicated the association of bromine in the polymer. The polymer obtained by this system was brownish in color and gave the test for bromine when the sample was dissolved in dil. $\mathrm{HNO}_{3}$ and tested with $\mathrm{AgNO}_{3}$ solution. The spot test ${ }^{15}$ with the polymer recovered from alcohol solution supported the inference. Estimation of bromine in PAN indicated that the termination was by molecular bromine in the experiment where $\left[\mathrm{Cu}^{2+}\right]:[\mathrm{DNPH}]$ was $1: 2$ (found $21.6 \%$, calculated $23.51 \%$ ), but the termination was due to combination when the ratio was $1: 4$ (found $7.69 \%$, calculated $7.73 \%$ ). Since some associated bromine are likely to escape during fusion, the amount of bromine was found always lower than the theoretical value. Therefore, bromine should be the inhibiting species ${ }^{16}$ for $2: 1$ experiment. It was found that the added bromine inhibits the polymerization. The following scheme may explain the reaction. Initiation must be due to tribromomethyl radical but an additional re- action takes place when the molar ratio of [DNPH] : $\left[\mathrm{Cu}^{2+}\right]$ is $2: 1$.

$$
\begin{aligned}
{\left[\mathrm{Cu}(\mathrm{DNPH})_{2}\right]^{2+}+\mathrm{CBr}_{4} \longrightarrow } & \mathrm{Cu}\left(\mathrm{CBr}_{2}\right)_{2} \\
& +\mathrm{Br}_{2}+\mathrm{DNPH} .
\end{aligned}
$$

The presence of bromine in the polymer was further confirmed from the far IR spectra of the poly(MMA) sample. A weak peak ${ }^{17}$ at $225 \mathrm{~cm}^{-1}$ indicated the formation of a CT complex between poly(MMA) radical and molecular bromine. The appearance of the strong peaks at $\sim 1730 \mathrm{~cm}^{-1}$ in IR and at $\sim 362 \mathrm{~cm}^{-1}$ in the far IR region indicated the incorporation of MMA in the polymer.

Since, bromine could be detected by UV spectroscopy, far IR and spot test, the inhibiting species should be bromine produced in situ. Estimation of bromine in the polymer indicates the presence of five bromine atoms, three due to end group $\mathrm{CBr}_{3}$ and two for the associated molecular bromine.

\section{REFERENCES}

1. S. Hussain, S. D. Baruah, and N. N. Dass, J. Polym. Sci., B, 16, 167 (1978).

2. S. D. Baruah and N. N. Dass, Eur. Polym. J., 16, 11 (1980).

3. S. D. Baruah and N. N. Dass, Makromol. Chem., 180, 1351 (1979).

4. S. Hussain and N. N. Dass, Eur. Polym. J., 18, 795 (1982).

5. S. R. Sen and N. N. Dass, Eur. Polym. J., 18, 477 (1982).

6. S. S. Begum and N. N. Dass, J. Polym. Sci., Polym. Lett. Ed., 20, 297 (1982).

7. S. S. Begum and N. N. Dass, J. Polym. Sci., Polym. Chem. Ed., 23, 2561 (1985).

8. A. K. Gogoi and N. N. Dass, J. Polym. Sci., Polym. Chem. Ed., 21, 3517 (1983).

9. S. Lenka and A. K. Dhal, J. Polym. Sci., Polym. Chem. Ed., 20, 587 (1982).

10. S. D. Baruah and N. N. Dass, Makromol. Chem., 182, 3591 (1981).

11. "Encyclopedia of Polymer Science and Technology," Vol. 1, Interscience, New York, N. Y., 1971, p 408.

12. N. C. Billingham, A. G. Chapman, and A. D. Jenkins, J. Polym. Sic., Polym. Chem. Ed., 18, 827 (1980).

13. S. D. Baruah and N. N. Dass, Eur. Polym. J., 17, 513 (1981). 
14. M. Watanabe and H. Kiuchi, J. Polym. Sci., 58, 103 (1962).

15. F. Feigl, "Spot test in Organic Analysis," 7th English Edition, Elsevier Publication Company, Amsterdam, London, New York, 1966, p 70.
16. M. K. Saha, M. Sen, and D. Pramanick, J. Polym. Sci., $A-1$, 4, 2137 (1966).

17. E. N. Gur'yanova, I. P. Gol'dshtein, and I. P. Romm, "Donor Acceptor Bond," John Wiley \& Sons, New York, N. Y., 1975, p 259. 